\title{
L'Elettrice Palatina: Mecenetismo, coleccionismo, formación y la misión del museo
}

\author{
L'Elettrice Palatina: mecenetismo, collectionism, training and \\ the The Museum's mission
}

\begin{abstract}
Anna Maria Luisa de' Medici. Elettrice Palatina ${ }^{1}$. Atti delle celebrazioni 20022004 Palazzo Vecchio Firenze. Firenze: Edizioni Polistampa, 2005. ${ }^{2}$
\end{abstract}

\author{
Anna Maria Luisa de Médici (Ludovica), princesa electora del Palatinado. Actas \\ de las celebraciones 2002-2004. Palazzo Vecchio Firenze (lugar: Palacio Viejo \\ de Florencia). Editorial Polistampa, 2005.
}

Franco Cavagnaro Farfán ${ }^{3}$

\section{RESUMEN}

El presente artículo es una reseña crítica y creativa sobre las actas de las celebraciones que, sobre su vida y legado, se realizaron entre el 2002 y el 2004 y que fueron publicadas en Firenze por la editorial Polistampa con el título: Anna Maria Luisa de' Medici elettrice palatina: atti delle celebrazioni, 2002-2004, Palazzo Vecchio, Firenze, bajo la coordinación de Anita Valentini.

La particularidad de este trabajo radica en el cruce epistemológico que se realiza para ver en ello el origen de los museos públicos, en el legado que la electora Anna Maria Luisa de' Medici realizó para que la colección medicea (de artes, libros, instrumentos científicos) no se disperse, ni sea acaparada por una familia o sea enajenada.

\section{Palabras clave}

Anna Maria Luisa de' Medici, Electora Palatina, coleccionismo privado, museos públicos, patrimonio cultural de Florencia, Patto di Famiglia

\section{ABSTRACT}

This article is a critical and creative review of the proceedings of the celebrations that, over his life and legacy, took place between 2002 and 2004 and which were published in Florence by the Polistampa publishing house with the title: Anna Maria Luisa de 'Medici elettrice palatina: atti delle celebrazioni, 2002-2004, Palazzo Vecchio, Firenze, under the coordination of Anita Valentini.

The particularity of this work lies in the epistemological crossing that takes place to see in it the origin of public museums, in the legacy that the elector Anna Maria Luisa de' Medici made so that the medicea collection (of arts, books, scientific instruments) did not disperse, nor was hoarded by a family or alienated.

\section{Keywords}

Anna Maria Luisa dei Medici, Palatine elector, private collection, publics museums, Florence cultural heritage, Patto di Famiglia

\footnotetext{
1 El llamado Palatinado, o más exactamente Condado Palatino del Rin, es el territorio que le pertenecía al Conde Palatino (Pfalzgraf), perteneciente al Sacro Imperio Romano-Germánico desde el siglo XIII y era un condado electoral. Políticamente se dividía en Alto Palatinado y Bajo Palatinado. Anna María Luisa de’ Medici es considerada como la última representante de la casta de los Médici y que marca un hito fundamental en la historia de Florencia (N. del E.).

2 El presente artículo trata sobre las actas a las que el subtítulo hace mención.

3 Licenciado en Literatura Hispánica por la Pontificia Universidad Católica del Perú. Ha publicado la novela El Viaje (Film de Treinta Minutos). Fue finalista en la Bienal de Novela Premio Copé 2011, con Me He Puesto el Traje Aquel. En 2016 publicó Huaquero (Una Historia sobre la Historia), la cual junto con la anterior conforman su Díptico del Pasado.
} 


\section{INTRODUCCIÓN}

Una fecha en especial: el 18 de febrero de 1743, señala la recordación de aquel día dedicado a Ana María Luisa de' Medici (1667 - 1743), Elettrice Palatina, cuyo mayor legado fue el Patto di Famiglia ${ }^{4}$ de 1737, sobre todo en su terzo articolo. De esta forma, la fecha de fallecimiento de Ana María Luisa se transforma en la Fiesta oficial de la Comune di Firenze. Con ello vino aparejada la publicación del libro que reseñamos aquí.

Dos aspectos de su vida noble nos ponen sobre aviso: su calidad de coleccionista, junto a su esposo, Johann Wilhelm von der Pfalz-Neuburg, con quien formaban la pareja electoral. Y su mecenetismo.

Se recuerda este Patto y este articolo por su cuidado y preclaridad (lungimiranza, en italiano) en salvaguardar el rico patrimonio familiar mediceo de la depredación por parte de la casa dinástica entrante, ajena a la ciudad de Firenze. Sin embargo, la Elettrice no lega, directamente, a Firenze ni a su pueblo la proprietá de su rica colección, sino que convierte en bienes inamovibles, inalienables, su colección, a la nueva dinastía reinante, los Ausburgo Lorena.

Al no contar con un hijo heredero, de la pareja electoral, la previa muerte del hermano mayor y el matrimonio fallido del segundo hermano, también sin hijos, dejan alos Medici sin heredero, ni candidato para el Granducato ${ }^{5}$. Cosimo III, padre de Ana Maria Luisa, propuso a su hija ante el Emperador como sucesora, pero esto no lo pudo conseguir, por ello la Elettrice, como último esfuerzo, luego de la muerte del esposo, y ya de regreso a Firenze, desde Düsseldorf, en 1717, decide salvaguardar, junto a importantes funcionarios de su ciudad natal, esos bienes. De ahí la redacción y el acuerdo de este importante documento.
Según Eugenio Giani, Assessore alla Valorizzazione delle Feste e Tradizioni Popolari del Comune di Fierenze, este complejo de bienes fue vinculado al Estado, que en aquel momento coincidía con Il Granducato di Toscana, uno de los antiguos estados preunitarios de la península italiana (antichi Stati preunaitari della penisola). Para Cristina Acidini Luchinat, comparándola con un conjunto de leyes italianas más contemporáneas (digamos aquellas entre 1939 y 2004), considera il Patto más potente.

Dunque, come é utile chiarire ancora una volta, l'Elettrice non dispose in favore della cittá di Firenze, né aurebbe potuto farlo essendo i Lorena leggitimamente subentranti al trono: e i successivi passaggi di proprietá dai Lorena ai Savoia a dai Savoia allo Stato italiano spiegano perché i musei e le biblioteche della cittá ove maggiormente si concentra il lascito mediceo siano di proprietá statale e non comunale. (p. 32$)^{7}$

Es importante esta distinción, pues es precisamente Giani, quien se encarga de establecer la distinción entre el Paese (con $\mathrm{P}$ mayúscula), Italia, y su reciente historia republicana unitaria de 150 años frente a los Estados Municipales italianas antes de la unificación (o paesi con $\mathrm{p}$ minúscula), cuya historia se remonta hasta el Medioevo, y todavía anteriores (e ancor prima) como señal de una identidad local fuerte. Sin embargo, para Mario Augusto Lolli, el Patto -cuya denominación en verdad es Convención (o Convenzione)- no trata solamente de la herencia artística de Casa Medici y de su conservación en Firenze, como comúnmente se cree. Establece sobre todo el rango, el tratamiento, los privilegios, los aposentos y los bienes de los que gozará

\footnotetext{
Pacto de familia.

5 Gran Ducado de Toscana (con sede en Florencia).

6 Asesor para la valoración de las fiestas y tradiciones populares de la Municipalidad de Florencia.

7 Las negritas son mías. Todas las traducciones del italiano al español son también mías. "Entonces, como es útil aclarar una vez más, l'Elettrice no decidió a favor de la ciudad de Florencia, ni habría podido hacerlo estando los Lorena legítimamente accediendo al trono: y los sucesivos cambios de propiedad de los Lorena a los Savoia y de estos al Estado italiano explican por qué los museos y las bibliotecas de la ciudad, donde mayormente se concentra el legado mediceo, sean de propiedad estatal y no comunal".
} 
la Elettrice, en el momento en que la nueva casa dinástica asuma el gobierno del Estado. Para el estudioso en el tercer artículo (terzo articolo, un pequeño codicillo): "si parla, notate bene, del patrimonio familiare: é la conservazione di una ereditá di familia" ${ }^{8}$. (p. 65)

\section{Il terzo articolo}

La Serenissima Elettrice cede, dá e trasferisce al presente a S.A.R. per Lui, e i Suoi Successori Gran Duchi, tutti i Mobili, Effetti e Raritá della successione del Serenissimo Gran Duca suo fratello, come Gallerie, Quadri, Statue, Biblioteche, Gioie ed altre cose preziose, siccome le Sante Reliquie e Reliquiari, e loro Ornamenti della Capella del palazzo Reale, che S.A.R si impegna di conservare, a condizione espresa che di quello [che] é per ornamento dello Stato, per l'utilitá dei cittadini e per attirare la curiositá dei Forestieri, non ne sará nulla trasportato, o levato fuori della Capitale, e dello Stato del Gran Ducato. (p. 11)

La forma en que Cristina Acidini Luchinat contextualiza el análisis de estas 3 frases esenciales, marcadas en negritas, vuelve al tema inicial de las Humanidades.

Para ello, Acidini nos pone sobre aviso acerca del siglo XVII:

Peró ricostruendo il clima settecentesco, in cui veramente l'aurora del Secolo dei Lumi si era giá manifestata, si potrebbe giá esprimere qualche considerazione. Per esempio, mi pare leggitimo affermare che "ornamento dello Stato" fosse un concetto piú proffondo e pregnante di quanto non ci appaia oggi [...] "Ornamento", invece, nel lenguaggio del passato significava la ragione stessa della bellezza morale o fisica, era un carattere intrinseco e non estrinseco, connaturato e non accesorio. (p. 34) ${ }^{10}$

Aún no estamos en el ambiente revolucionario de la Francia de 1789. Y no obstante, las luces del conocido como Siglo de las Luces ya alcanzan sus primeros esplendores. Es el Illuminismo (la ilustración), tal y como se le conoce en Italia. El segundo término a analizar es el de utilitá. Según Acidini la presencia de la colección medicea en Firenze debía ser interpretada como un factor de formación del temperamento, de gusto, de capacidad crítica para todos los ciudadanos. En mi opinión este es un tema esencial también de la propia época: formación como valor intrínseco, y luego la formación del lado estético como fin último del aprendizaje.

Finalmente, la curiositá dei Forestieri, se refiere al inicio todavía soterrado, pero pleno de potencialidad, de la llegada de los hombres y mujeres del Grand Tour

"che con un certo spirito di avventura varcavano le Alpi e si portavano da una capitale all'altra nell'ininterroto percoso artistico che era l'Italia del primo Settecento. Ella, con la sua visione, aveva anticipato quello che sarebbe stato lo suiluppo del

\footnotetext{
"se habla, nótese bien, del patrimonio familiar: es la conservación de una herencia y heredad de familia".

9 Las negritas son mías. "La Serenísima Electora cede, da y transfiere al presente a S.A.R. para Él, y Sus Sucesores Grandes Duques, todos los Muebles, Efectos y Rarezas (curiosidades) de la sucesión (patrimonio) del Serenísimo Gran Duque a su hermano, como Galerías, Cuadros, Estatuas, Bibliotecas, Joyas y otras cosas preciosas, así como las Santas Reliquias y relicarios, y sus Ornamentos de la Capilla del Palacio Real, que S.A.R se empeña en conservar, a condición expresa que aquello ŚqueȘ es para ornamento del Estado, para la utilidad de sus ciudadanos y para atraer la curiosidad de los Forasteros, no sea nunca transportado, o llevado fuera de la Capital, y del Estado del Gran Ducado".

10 Las negritas son mías. "Pero reconstruyendo el clima del siglo XVIII, en el que verdaderamente el amanecer del Siglo de las Luces se había, ya, manifestado, se podría expresar alguna consideración. Por ejemplo, me parece legítimo afirmar que "ornamento dello Stato" (ornamento del Estado) fuese un concepto más profundo y cargado de significado de lo que nos parece hoy [...] "Ornamento", en cambio, en el lenguaje del pasado significaba la razón misma de la belleza moral o física, era un carácter intrínseco y no extrínseco, connatural y no accesorio".
} 
turismo d'arte: prima elitario, poi di massa, decisivo nel destino della cittá"11. (p. 35)

\section{Illuminismo y Grand Tour: la comprensión del patrimonio a través de lo estético}

Goethe viaja a Italia en 1786.

Lo hace como parte de su aprendizaje (aprendistato) sobre la belleza clásica que cree encontrará en las grandes ciudades italianas. La Elettrice ha muerto y el Patto di Famiglia ya fue firmado, la colección de objetos de arte salvados de los Ausburgo Lorena. Sin embargo, demos algunos detalles importantes, ahora, acerca de la Ilustración (Illuminismo) y el Grand Tour.

Goethe viaja por varios motivos. Apenas ha terminado su primera versión de Los años de aprendizaje de Wilhelm Meister, conocida como la Sendung. En una carta escrita al Conde Augusto dice:

\section{"La principal atención que abrigaba en mi viaje era curarme de los males físicos y morales que me torturaban en Alemania y que me habían inutilizado, además quería calmar una sed ardiente de arte verdadero. Lo primero solo lo he conseguido medianamente, lo segundo de un modo pleno".}

En estos años previos a la Revolución Francesa, el ideal de la formación era el centro de las discusiones filosóficas y será éste aún más aguzado en la obra de Goethe, Los años de aprendizaje. El término italiano "iluminista" viene precisamente de este deseo de superar la oscuridad a través de la razón. "La idea de la educación estética como piedra de toque de la auténtica formación es una idea-guía de la época, recuérdense a este respecto las Cartas para la educación estética del hombre (1793/1795) de Schiller" (Salmerón, p. 28) ${ }^{12}$.
Por eso tantos términos acuñados durante este periodo, como, por ejemplo: Bild (entendido como formación), que a su vez forma el Bildungsroman (novela de aprendizaje), son totalmente claves en la tradición autoformativa del siglo XVIII. "Este concepto, como todos los conceptos guía de esta tradición, es fruto del intento de superar el hiato individualidadobjetividad y así armonizar los dos atributos que debe tener el movimiento formativo" (Salmerón, p. 48).

Apreciar la belleza clásica se transforma así en un viaje iniciático, y hacerlo a través de la experiencia directa in situ. Los jóvenes adinerados van desde Inglaterra, primeramente, hacia Italia, pasando por Francia. Luego también lo hacen desde Alemania, como en el caso de Goethe. Ese paso tendrá una honda huella en la mejor literatura alemana, que va desde Goethe hasta Thomas Mann. Visto de esta forma Italia se presentará en la tradición alemana como lo exótico, lo cálido y lo irracional. Y de algún modo, incumbe este artículo, pues es un viaje contrario el de Goethe al de la Elettrice Palatina: pasa desde Il Gran Ducato hasta la ciudad de su esposo, Düsseldorf, para regresar a Florencia, tras la muerte del esposo.

No obstante, para Cecilia Filippini, Storica dell'Arte ${ }^{13}$, 1'Illuminismo de la Elettrice, se puede apreciar en el lema que aparece en muchas de sus medallas: A Deo omnia. Al mismo tiempo, este motto se correspondía con el emblema de un sol radiante y la inscripción diffuso lumine. Palabras que en consideración de la autora anticipan su preclaridad ${ }^{14}$ (lungimiranza), la cual se sistematizará en la prevención que se tomará en el inventario de los bienes mediceos, luego en la conservación y finalmente en la consciencia (conscenza) del patrimonio artístico. Esto se puede relacionar a su vez con los criterios programáticos de la cultura settecentesca, y que también son parte de la tradición de la cultura italiana.

\footnotetext{
11 ... que con un cierto espíritu de aventura cruzaban los Alpes y se trasladaban de una capital a otra en el ininterrumpido recorrido artístico que era Italia de la primera parte del siglo XVIII. Ella, con su visión, había anticipado aquello que sería el desarrollo del turismo del arte: primero elitista, después masivo, decisivo en el destino de la ciudad.

12 Las citas se encuentran en la edición traducida por Miguel Salmerón Los años de aprendizaje de Wilhelm Meister de Johann Wolfgang Von Goethe. Ediciones Cátedra, Madrid: 2000

13 Historiadora del Arte.

14 Pero, también, precaución.
} 


\section{La misión del museo}

Al respecto, es precisamente Cristina Acidini quien hace una diferenciación geográfica $e$ idiosincrática acerca de la misión del museo, tomando la dualidad del norte y el sur.

Quedó fascinada por su diferencia entre el museo italiano y aquel del mundo anglosajón (anglosassone).

En Italia ha nacido el museo moderno $y$, al mismo tiempo, sus ciudades son precisamente museos al aire libre, en cada iglesia, en cada antigüedad (antichitá) romana hay arte y belleza.

Il museo é concepito come una sorta di Arca di Noé che racchiude le testimonianze del passato $e$ naviga, attraverso i rischi dell'oggi, verso un futuro in cui questi beni saranno consegnati alle prossime generazioni (p. 33) ${ }^{15}$.

Esta imagen me pareció muy pertinente. En reciente visión del film documentario de Alexander Sukorov, Francophonia (2015), inmerso como figura dentro de la ficción, el director entabla un diálogo con un amigo que contrariado dirige un carguero (repleto del mejor arte) que atraviesa un mar embravecido, y se queja, puja, enfrenta, pierde la comunicación por su lejanía con otro interlocutor ajeno a su tarea (Sukorov). Aunque es el director quien nunca se mueve de su habitación, solo es reflexión y voz en off a través de las galerías. El film muestra la historia del Museo de Louvre, centrado sobre todo en la disputa por la conservación de sus obras durante la ocupación nazi de París.

Esta imagen del carguero en el mar, me sugería también la del Arca de Noé, asediada por el tiempo, las guerras, los robos, las conspiraciones, los cambios de temperatura en los mares, los desastres climáticos próximos, simbolizados por el mar, y, al mismo tiempo, tenía resonancias en el pasado posterior al Settecento con los trofeos de guerra napoleónicos que se consignan en algunos de los artículos del libro en reseña y que, tal como Sukorov ficcionaliza, algunos de ellos se hundieron y perdieron en el mar. Una época de coleccionismo imperial.

Por otro lado, el museo anglosassone se ha formado con colecciones privadas: il museo concepito come un servicio a vantaggio della collettivitá per scopi ben precisi. ${ }^{16}$ Es decir, un servicio en beneficio de la colectividad.

La primera misión trata de ser un receptáculo, un empréstito temporal, cuya misión es llevar el testimonio a las generaciones futuras. La segunda refuerza el sentido formativo (me arriesgaría a pensar en una narrativa didáctica que guíe al desmemoriado y virginal espectador, consciente-inconsciente de los devenires de la historia) en búsqueda de la formación, el Bild. Precisamente, el mismo Bild del Grand Tour. Al parecer son contrarias. Pero, en verdad, parecen más bien complementarias. La primera simplemente desea presentar las reliquias del pasado, salvaguardadas del tiempo, las guerras, los robos, las suplantaciones, mientras que la otra pareciera buscar una explicación del pasado y entregarla al despistado y afásico espectador del presente. El ejemplo de la posición mediterránea es la propia vida de la Elettrice. El terzo articolo del Patto di Famiglia: el epílogo de una era, que busca salvaguardar el legado familiar ante la llegada de una nueva era, una nueva dinastía.

Finalmente, en el testimonio de Christian Constantini, Comandante del Nucleo Carabinieri per la Tutela del Patrimonio Culturale di Firenze, encontramos una concordancia con el esfuerzo de la Elettrice en la protección y devolución de las piezas italianas a su lugar de origen cuando éstas han sido robadas y trasladadas al extranjero. La creación de este Comando Carabinieri per la Tutela del Patrimonio Culturale data de 1969, un año antes de la Convención de París de 1970. Su database computarizada fue

\footnotetext{
15 "El museo es concebido como una suerte de Arca de Noé que contiene los testimonios del pasado y navega, a través de los riesgos del hoy, hacia el futuro en el que estos bienes serán entregados a las próximas generaciones".

16 "El museo es concebido como un servicio para ventaja de la colectividad con objetivos bien precisos".
} 
desarrollada diez años después, tan temprano como 1980. Para encontrar una obra en esa database, es importante que ésta ya esté catalogada, que se tenga una foto de ella y que de ésta se tenga conocimiento de datos precisos. La catalogación es un instrumento importantísimo.

Fotografare significa avere una traccia indelebile di un'opera che puó essere di qualsiasi natura [...] lo Stato italiano interviene nella tutela delle opere d'arte create nella Penisola dall'uomo e dalla natura. (p. 90) ${ }^{17}$

Existe, entonces, además de la preocupación por artículos como los que estuvieron en posesión de la Elettrice Palatina, una por el paesaggio y los bienes culturales de la antichitá.
Finalmente todo el esfuerzo del inventario está bien sopesado por Cecilia Filippini para el caso precedente de la Elettrice:

Ora tutti noi siamo consapevoli dell'importanzadegliinventari,come primo passo per la catalogazione $e$, di conseguenza, per la tutela appunto del patrimonio artistico: ma a quel tempo, nella prospettiva a breve termine, era un tentativo per difendere i gioilli e le preziose suppelletili dalla rapacitá dei funzionari poco onesti dipendenti dai Lorena, a lungo termine un utile strumento di valutazione per consentiré a noi posteri l'esatta percezione del collezionismo degli ultimi Medici, in rapporto anche a quello dei loro antenati. (p. 87) $)^{18}$

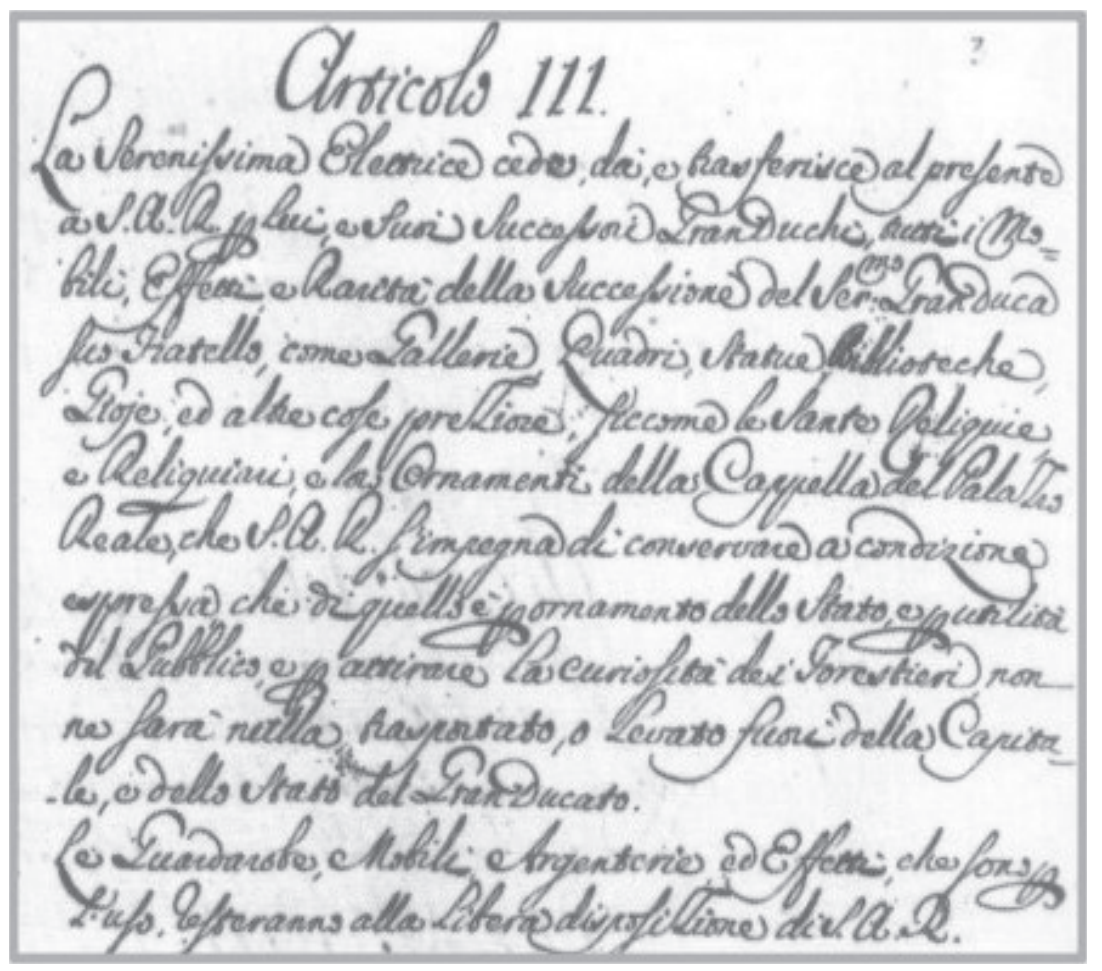

Convenzione di Famiglia (Vienna 1737) - Artícolo III - Traduzione in Italiano dell'originale in francese

Fuente: https://www.unifi.it/upload/sub/Anna_Maria_Luisa_Medici_31-10-17.pdf

\footnotetext{
17 "Fotografiar significa tener una huella indeleble de una obra que puede ser de cualquier naturaleza [...] el Estado italiano interviene en la tutela de las obras de arte creadas en la Península por el hombre y la naturaleza”.

18 "Ahora todos somos conscientes de la importancia de los inventarios, como primer paso para la catalogación y, en consecuencia, para la tutela precisamente del patrimonio artístico: pero en aquel tiempo, con una perspectiva de breves miras, era un tentativo para defender las joyas y los preciosos adornos de la rapacidad de los funcionarios poco honestos dependientes de los Lorena, y con una perspectiva de larga mira, un útil instrumento de valoración para permitirnos a los posteriores la exacta percepción del coleccionismo de los últimos Medici, en relación también a aquel de sus antepasados".
} 


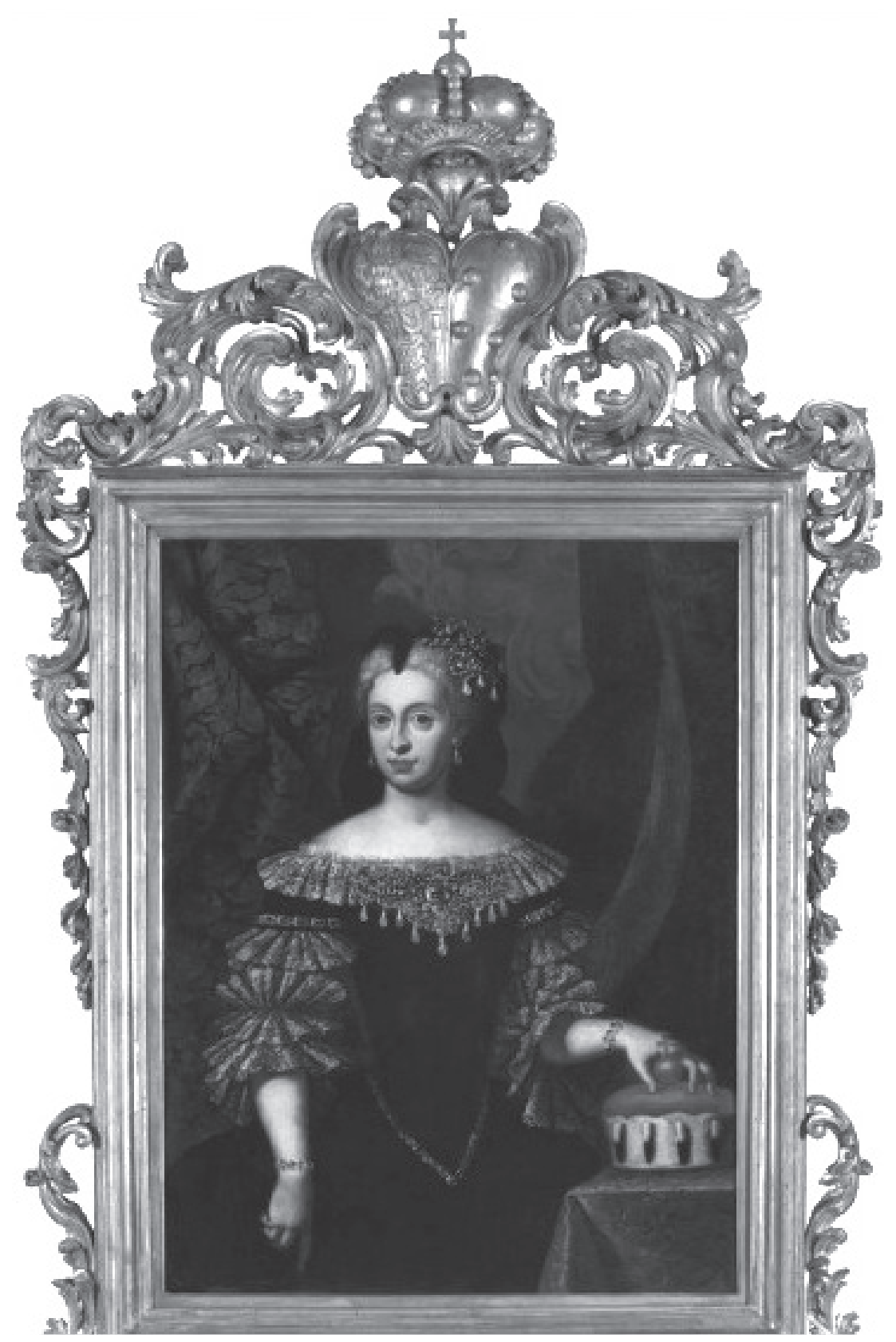

L'Elettrice Palatina Anna Maria dei Medici (Colezione d'Arte Fondazione CR Firenze. Fuente:https://www.unifi.it/upload/sub/Anna_Maria_Luisa_Medici_31-10-17.pdf 


\section{REFERENCIAS}

Valentini, Anita (2005). Anna Maria Luisa de' Medici elettrice palatina: atti delle celebrazioni, 2002-2004, Palazzo Vecchio, Firenze. Firenze, Italia. Polistampa.

Fecha de recepción: 22-03-18

Fecha de aceptación: 04-05-18 